\title{
Classification of Covariance Matrix
}

\section{Eigenvalues in Polarimetric SAR for Environmental Monitoring Applications}

Pia Addabbo, Member, IEEE, Filippo Biondi, Member, IEEE, Carmine Clemente, Senior Member, IEEE, Danilo Orlando, Senior Member, IEEE, and Luca Pallotta, Senior Member, IEEE

\begin{abstract}
In this paper, we describe novel techniques for automatic classification of the dominant scattering mechanisms associated with the pixels of polarimetric SAR images. Specifically, we investigate two operating scenarios. In the first scenario, it is assumed that the polarimetric image pixels locally share the same covariance (homogeneous environment), whereas the second scenario considers polarimetric pixels with different power levels and the same covariance structure (heterogeneous environment). In the second case, we invoke the Principle of Invariance to get rid of the dependence on the power levels. For both scenarios, we formulate the classification problem in terms of multiple hypothesis tests which is addressed by applying the model order selection rules. The performance analysis is conducted on both simulated and measured data and demonstrates the effectiveness of the proposed approach.
\end{abstract}

\section{Index Terms}

Coherence and covariance matrix, eigenvalues decomposition, model order selection rules, polarimetric SAR image classification, RADARSAT-2 quad-polarization SLC.

Pia Addabbo is with Università Telematica "Giustino Fortunato", via Delcogliano 12, 82100, Benevento, Italy.

Filippo Biondi is with Italian Ministry of Defence. E-mail: biopippo@gmail.com.

Carmine Clemente is with the University of Strathclyde, Department of Electronic and Electrical Engineering, 204 George Street, G1 1XW, Glasgow, Scotland.

Danilo Orlando is with the Faculty of Engineering, Università degli Studi "Niccolò Cusano", 00166 Roma, Italy. E-mail: danilo.orlando@unicusano.it.

Luca Pallotta is with CNIT udr Università "Federico II", via Claudio 21, I-80125 Napoli, Italy. E-mail: luca.pallotta@unina.it. 


\section{INTRODUCTION}

The use of Polarimetry in the context of Synthetic Aperture Radars (SARs) is gaining more and more interest from the scientific community as well as from companies developing commercial products using techniques exploiting this sensing mode. The main benefit of the use of polarimetry resides in the provision of additional information about the scattering properties of the illuminated scene or target, allowing for significant performance improvements in both civilian and military applications [1]-[5]. The exploitation of SAR polarimetry is of particular relevance in Remote Sensing. In this context, extended area monitoring and target areas classification receive the widest attention by researchers. A nonexhaustive list of applications of polarimetric SAR in remote sensing includes biomass estimation, rice paddy monitoring, snow and ice analysis, oil spill detection and land-use classification. In [6] data from both SIR-C and X-SAR sensors were used to estimate biomass characteristics with relatively small error. The capability of polarimetric SAR data to provide information about rice phenology was demonstrated in [7], where data from TerraSAR-X were used to assess test rice paddy in Spain. The problem of assessing snow wetness was addressed in [8], in which the authors proposed a polarimetric model to obtain the relationship between snow wetness and the polarization and demonstrated it through SIR-C/X-SAR data. In [3], the problem of oil spill detection was addressed through the use of polarimetric covariance symmetries, while in [9] a similar framework was used to define a novel polarimetric classification method to support land-use analysis. In the classification context, the information extracted from the polarimetric channels is typically used to characterize different zones of the acquired scene in an automatic or semi-automatic way [9]-[13]. A common approach relies on the manipulation and decomposition of the so-called Polarimetric Covariance Matrix (PCM) computed from the vectorized form of the Sinclair matrix [10, pag. 63]. It contains hidden information to discriminate different [14]:

- areas in the SAR image, e.g., land vs sea, bare vs cultivated fields, buildings vs trees;

- categories of the same typology, e.g., small stem crops vs broad leaves crops;

- scattering mechanisms, e.g., dipole vs diplane vs trihedral, and so on.

Generally speaking, existing strategies for scene classification from SAR images can be grouped into two main categories, namely unsupervised and supervised algorithms, and have been extensively investigated [9], [15]-[24]. The former consists of clustering image pixels by means of common characteristics/features and occurs in an automatic way without any kind of aid from the user. On the other hand, the latter exploits training pixels, which are a-priori selected by the user to define the features identifying a specific class. Now, image classification can be accomplished by means of PCM decomposition. In fact, several 
strategies have been proposed in scientific literature [10], [14], [25]. For example, in [12], a robust framework for polarimetric SAR covariance symmetries classification was derived and applied to L-Band data (Figure 1).

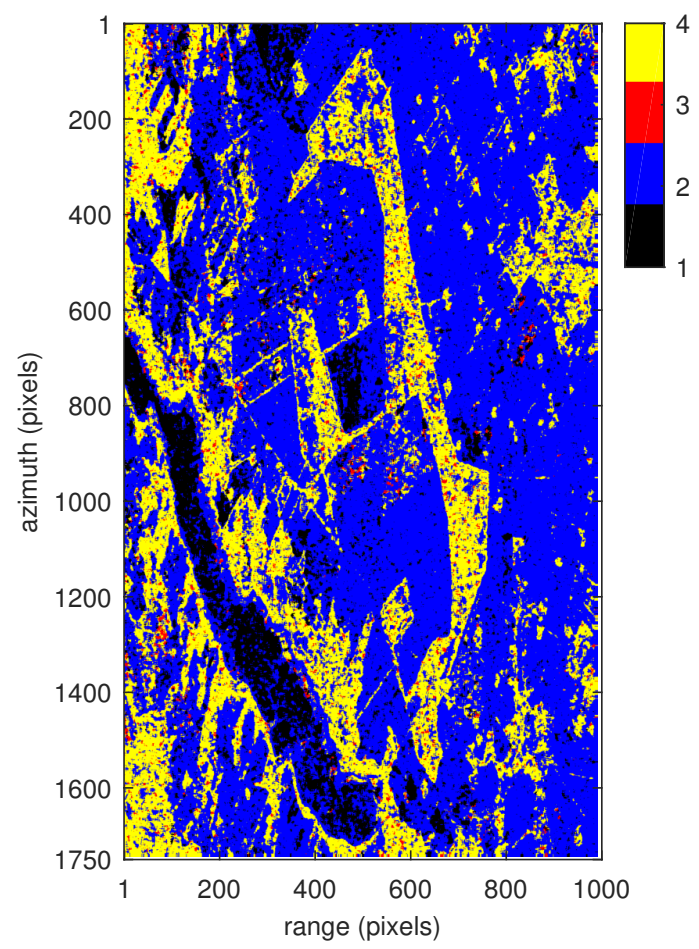

Fig. 1. L-Band polarimetric SAR data classified using the robust approach presented in [12].

The same principle has also been extended to the Polarimetric Interferometric SAR (PolInSAR) scenario in [13], where the additional elevation information has been exploited to map the symmetries in 3D as shown in Figure 2.

In addition, in [9] the polarimetric covariance symmetries after being classified are used to extend the $H / \alpha$ Polarimetric Classification in the Symmetric $H / \alpha$ Polarimetric Classification method, as illustrated in Figure 3.

Among the different PCM decomposition approaches, the eigendecomposition allows gathering information about the scene reflectivity at different polarizations. Note that the PCM can be written as the non-coherent sum of three rank-one matrices associated with each eigenvector whose intensity is ruled by the corresponding eigenvalue (namely, each of them is characterized by a single scattering contribution) [10], [14]. Now, if only one eigenvalue is non-zero, then the covariance is related to a single scattering 

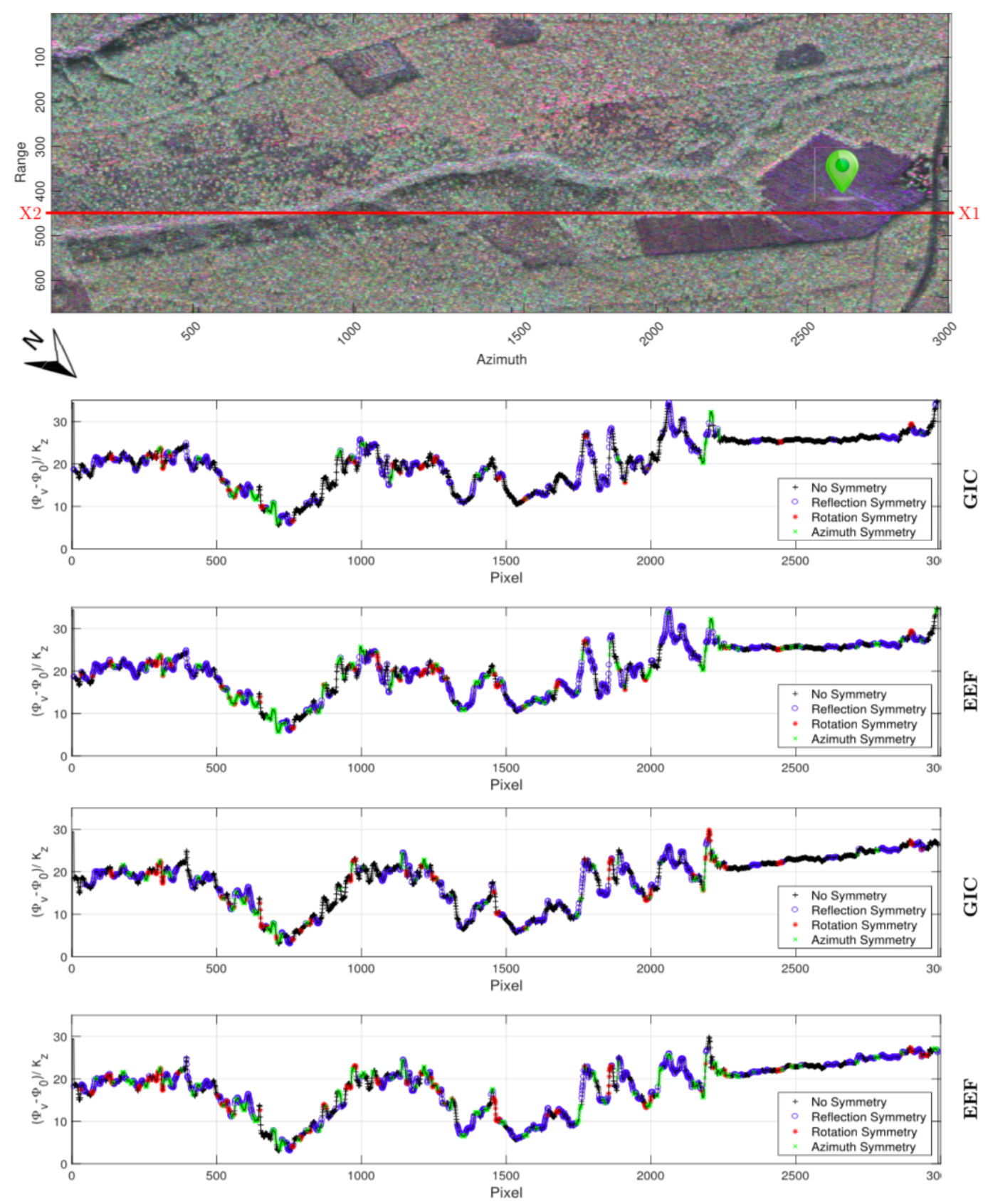

Fig. 2. PolInSAR covariance symmetries classification of BioSAR II L-Band data [13].

mechanism (this is the case of a pure target). On the contrary, if all eigenvalues are non-zero and share the same value, then the target corresponds to a non-polarized random scattering structure. Finally, other cases are possible and represent the situations where partially polarized scatterers are present and the resulting PCM exhibits non-zero and non-equal eigenvalues, i.e., two dominant eigenvalues or three 

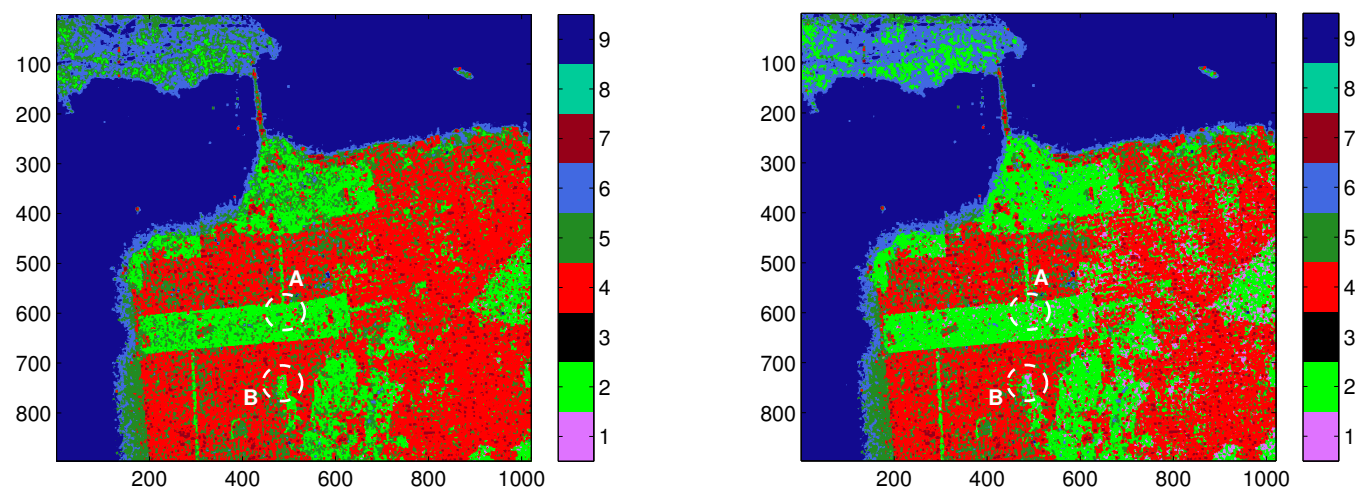

Fig. 3. Results from [9] on L-Band AirSAR data with the $H / \alpha$ classification (left) and the symmetric $H / \alpha$ classification (right) methods.

different eigenvalues. More details and insights can be found in [10], [14]. In [26], a recent work from some co-authors of the present paper, the problem of estimating the number of dominant covariance eigenvalues in polarimetric SAR images was investigated, focusing only on the heterogeneous scenario wherein the polarimetric image pixels share the same covariance but different power levels. In this paper, a more general framework is presented and assessed on different datasets. Specifically, given the pixel under test, the number of dominant eigenvalues for the pixel under test is classified exploiting the pixels in its neighborhood (i.e., looks) assuming that the same polarimetric covariance matrix is shared among the pixels (homogeneous environment) or, due to reflectivity variations of the scene, the covariance matrix exhibits different scaling factors from pixel to pixel (heterogeneous environment). These scaling factors are representative of the power levels raising from different backscattering strength [12]. In the latter case, original data are replaced by a Maximal Invariant Statistic (MIS) to remove the dependence on the nuisance parameters. Moreover, the statistical characterization of the MIS is provided along with suitable estimates of the unknown parameters. For both homogeneous and heterogeneous environment, the classification problem is, then, formulated as a multiple hypotheses test with nested hypotheses [27]. It is important to observe that in the presence of nested hypotheses, the classical Maximum Likelihood Approach (MLA) might return erroneous classifications. For this reason, the Model Order Selection (MOS) rules are used to synthesize classification architectures. Finally, the illustrative examples, built up using both simulated and measured RADARSAT-2 SAR data, show the effectiveness and usefulness of the proposed algorithms.

The remainder of this paper is organized as follows: Section II provides some preliminary definitions, 
while Section III is devoted to problem formulation, covariance estimation under each hypothesis, and the design of the MOS rules. In Section IV, the behavior of the proposed algorithms is assessed adopting the probability of correct classification as the performance metric. Finally, Section V draws conclusions and traces future researches.

\section{A. Notation}

The adopted notation uses boldface for vectors $\boldsymbol{a}$ (lower case) and matrices $\boldsymbol{A}$ (upper case). $\mathbb{C}^{N \times M}$ is the set of complex matrices of size $N \times M$ (or vectors if $M=1$ ). A diagonal matrix whose nonzero entries are the components of a vector $\boldsymbol{a}$ is denoted by Diag $(\boldsymbol{a})$. The transpose and the conjugate transpose are denoted by $(\cdot)^{T}$ and $(\cdot)^{\dagger}$, respectively. $\operatorname{tr}\{\cdot\}$ and $\operatorname{det}(\cdot)$ are the trace and the determinant of the square matrix argument, respectively. $\boldsymbol{I}$ denotes the identity matrix, whose size is determined from the context, and $\|\cdot\|$ denotes the Euclidean vector norm.

\section{Parameters Definition and Datacube Construction}

A multi-polarization SAR sensor, for each pixel of the image under test, measures $N=3$ complex returns for each range-azimuth resolution cell, which are collected from three different polarimetric channels (namely $\mathrm{HH}, \mathrm{HV}$, and $\mathrm{VV}$ ). The $N$ returns associated with the same pixel are organized in the specific order $\mathrm{HH}, \mathrm{HV}$, and $\mathrm{VV}$ to form the 3 -dimensional vector $\boldsymbol{x}_{l, m} \in \mathbb{C}^{3 \times 1}, l=1, \ldots, L$ and $m=$ $1, \ldots, M$ ( $L$ and $M$ are the vertical and horizontal size of the image, respectively). Therefore, the sensor provides a 3-D data stack $\boldsymbol{X}_{0}$ of size $M \times L \times N$ which is referred to in the following as datacube (see Fig. 4). Starting from the datacube of the illuminated scene, for the generic pixel under test, a rectangular neighbourhood $\mathcal{A}$ of size $K=W_{1} \times W_{2} \geq N$ is extracted. Finally, let $\boldsymbol{X}=\left[\boldsymbol{x}_{1}, \ldots, \boldsymbol{x}_{K}\right] \in \mathbb{C}^{3 \times K}$ be a matrix whose columns are the vectors of $\mathcal{A}$.

\section{Problem Formulation And Classification Strategies}

The polarimetric returns $\boldsymbol{x}_{1}, \ldots, \boldsymbol{x}_{K}$ are modeled as zero-mean circularly symmetric complex Gaussian vectors whose covariance matrix is $\sigma_{k}^{2} \boldsymbol{C}, k=1, \ldots, K[12]$. Thus, the probability density function (pdf) of $\boldsymbol{x}_{k}$ can be written as

$$
f\left(\boldsymbol{x}_{k} ; \sigma_{k}^{2}, \boldsymbol{C}\right)=\frac{1}{\left(\pi \sigma_{k}^{2}\right)^{3} \operatorname{det}(\boldsymbol{C})} \exp \left\{-\sigma_{k}^{-2} \operatorname{tr}\left[\boldsymbol{C}^{-1} \boldsymbol{x}_{k} \boldsymbol{x}_{k}^{\dagger}\right]\right\}
$$

Observe that the above expression encompasses the homogeneous environment (when $\sigma_{1}^{2}=\ldots=\sigma_{K}^{2}=$ $\sigma^{2}$ ) and the heterogeneous environment (when $\sigma_{i}^{2} \neq \sigma_{k}^{2}, \forall i \neq k$ ). In what follows, for the sake of simplicity and without loss of generality, we assume that $\sigma^{2}=1$. 

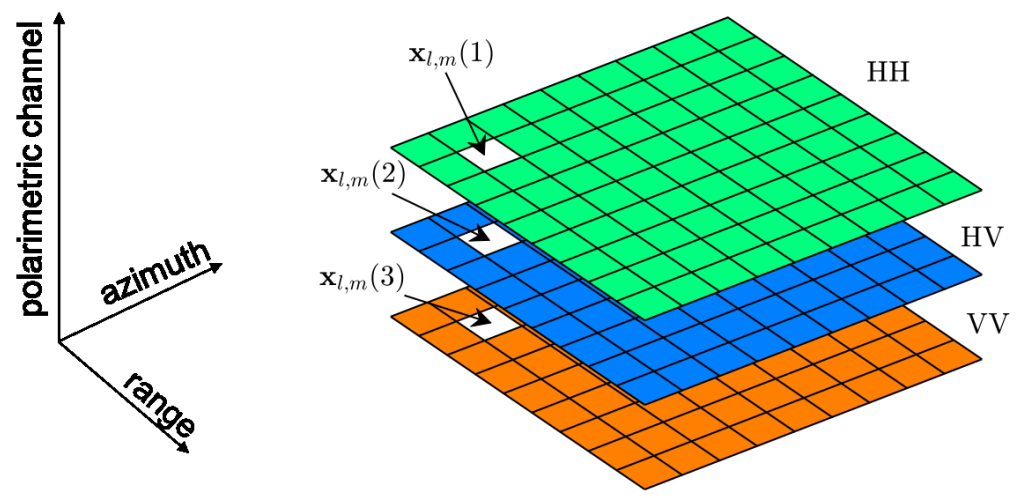

Fig. 4. Datacube for polarimetric images.

Before proceeding with the derivations, let $\boldsymbol{C}=\boldsymbol{U} \boldsymbol{\Lambda} \boldsymbol{U}^{\dagger}$ be the eigendecomposition of $\boldsymbol{C}$, where $\boldsymbol{\Lambda}=\operatorname{Diag}\left(\left[\begin{array}{lll}\lambda_{1} & \lambda_{2} & \lambda_{3}\end{array}\right]^{T}\right)$ is the diagonal matrix containing its eigenvalues arranged in decreasing order $\left(\lambda_{1} \geq \lambda_{2} \geq \lambda_{3}\right)$, and $\boldsymbol{U} \in \mathbb{C}^{3 \times 3}$ is a unitary matrix containing the corresponding eigenvectors. Now, the problem of eigenvalue pattern classification can be expressed as the following multiple hypotheses test:

$$
\left\{\begin{array}{l}
H_{1}: \lambda_{1}=\lambda_{2}=\lambda_{3}=\lambda \\
H_{2}: \lambda_{1} \geq \lambda_{2}=\lambda_{3} \\
H_{3}: \lambda_{1}=\lambda_{2} \geq \lambda_{3} \\
H_{4}: \lambda_{1} \geq \lambda_{2} \geq \lambda_{3}
\end{array}\right.
$$

Note that a "dominant" scattering behavior is associated with each image pixel on the basis of the specific pattern of the covariance eigenvalues. Moreover, the covariance is estimated resorting to the pixels belonging to $\mathcal{A}$.

Problem (2) contains nested hypotheses and, hence, the MLA might fail since the likelihood function monotonically increases with the number of unknown parameters. As a result, MLA would return always $H_{4}$, that is the hypothesis with the maximum uncertainty [28]. For this reason, the so-called MOS rules [29], which moderate the overfitting inclination of the compressed likelihood in the case of nested hypotheses, are involved in the design of classification architectures. Most of the MOS rules consist of a fitting term plus a penalty term (a point better explained in the next section). The former is a function of the compressed likelihood function, where the unknown parameters are replaced by their Maximum Likelihood Estimates (MLEs). Thus, it is required to provide the MLE expression of $C$ under the considered hypotheses. 
The herein considered MOS rules are the Akaike Information Criterion (AIC), the Generalized Information Criterion (GIC), and ${ }^{1}$ the Bayesian Information Criterion (BIC) [29]. The generic structure of the MOS rules exhibits the following form

$$
H_{\hat{i}}=\underset{\substack{H_{i} \\ i=1, \ldots, 4}}{\arg \min }\left\{-2 \mathcal{L}\left(\widehat{\boldsymbol{C}}_{i} ; \boldsymbol{X}\right)+p_{i}\right\}
$$

where $p_{i}=k_{p}(i) \eta$ is the penalty term, with $k_{p}(i)$ the number of unknown parameters under the $H_{i}$ hypothesis, and $\widehat{\boldsymbol{C}}_{i}$ is the MLE of $\boldsymbol{C}$ under $H_{i}$. The factor $\eta$ takes on the following values

$$
\eta= \begin{cases}2, & \text { AIC } \\ 1+\rho, \rho \geq 1, & \text { GIC } \\ \log K, & \text { BIC }\end{cases}
$$

\section{A. Homogeneous Environment}

As stated before, under the homogeneity assumption, the pixel belonging to the neighbourhood of that under test share exactly the same covariance matrix. As a consequence, the resulting log-likelihood can be written as

$$
\mathcal{L}(\boldsymbol{C} ; \boldsymbol{X})=-3 K \log \pi-K \log \operatorname{det}(\boldsymbol{C})-\operatorname{tr}\left[\boldsymbol{C}^{-1} \boldsymbol{S}\right]
$$

where $\boldsymbol{S}=\boldsymbol{X} \boldsymbol{X}^{\dagger}$. Now, observe that classifier (3) exploits the compressed log-likelihood where $\boldsymbol{C}$ is replaced by its MLE. To this end, in the proposition below the expressions of these estimates are computed.

Proposition 3.1: The estimates of $\boldsymbol{C}$ under the $H_{i}$ hypothesis, $\widehat{C}_{i}$ say, and the resulting compressed log-likelihoods can be computed as follows.

1) Under $H_{1}$ : it is not difficult to show that log-likelihood can be recast as

$$
\mathcal{L}(\boldsymbol{C} ; \boldsymbol{X})=c-3 K \log \lambda-\frac{1}{\lambda} \operatorname{tr}[\boldsymbol{S}]=\mathcal{L}(\lambda ; \boldsymbol{X}),
$$

where $c$ is a constant. Setting to zero the first derivative of $\mathcal{L}(\lambda ; \boldsymbol{X})$ with respect to $\lambda$ leads to

$$
\frac{d}{d \lambda}[\mathcal{L}(\lambda ; \boldsymbol{X})]=-3 K \frac{1}{\lambda}-\frac{1}{\lambda^{2}} \operatorname{tr}[\boldsymbol{S}]=0
$$

\footnotetext{
${ }^{1}$ The Embedded Exponential Family (EEF) is another MOS rules useful to solve the nested hypotheses. In this paper, it has been neglected because this decision criterion requires the computation of the Generalized Likelihood Ratio Test (GLRT) implying that a suitable $H_{0}$ hypothesis must be defined.
} 
namely

$$
\widehat{\lambda}=\frac{1}{3 K} \operatorname{tr}[S] .
$$

Thus, the compressed log-likelihood is given by

$$
\mathcal{L}\left(\widehat{\boldsymbol{C}}_{1} ; \boldsymbol{X}\right)=c-3 K \log \left\{\frac{\operatorname{tr}[\boldsymbol{S}]}{3 K}\right\}-3 K
$$

2) Under $H_{2}$ : let $\lambda_{23}=\lambda_{2}=\lambda_{3}$ and recast the log-likelihood as follows

$$
\begin{aligned}
\mathcal{L}(\boldsymbol{C} ; \boldsymbol{X}) & =c-3 K \log \left(\lambda_{1} \lambda_{23}^{2}\right)-\operatorname{tr}\left\{\boldsymbol{U D i a g}\left(\left[1 / \lambda_{1}, 1 / \lambda_{23}, 1 / \lambda_{23}\right]\right) \boldsymbol{U}^{\dagger} \boldsymbol{S}\right\} \\
& =\mathcal{L}\left(\boldsymbol{U}, \lambda_{1}, \lambda_{23}, \boldsymbol{X}\right)
\end{aligned}
$$

Thus, maximization over $\boldsymbol{C}$ is tantamount to the following problem

$$
\max _{\boldsymbol{U}} \max _{\lambda_{1}, \lambda_{23}} \mathcal{L}\left(\boldsymbol{U}, \lambda_{1}, \lambda_{23}, \boldsymbol{X}\right)
$$

Now, exploiting Theorem 1 of [30], it can be shown that

$$
\arg \max _{\boldsymbol{U}} \mathcal{L}\left(\boldsymbol{U}, \lambda_{1}, \lambda_{23}, \boldsymbol{X}\right)=\boldsymbol{U}_{S} e^{j \theta},
$$

where $\theta \in\left[\begin{array}{ll}0 & 2 \pi\end{array}\right]$ and $\boldsymbol{U}_{S} \in \mathbb{C}^{3 \times 3}$ is the unitary matrix containing the eigenvectors of $\boldsymbol{S}$. As a consequence, the partially compressed log-likelihood becomes

$$
c-K \log \lambda_{1}-2 K \log \lambda_{23}-\operatorname{tr}\left\{\boldsymbol{D i a g}\left(\left[\gamma_{1} / \lambda_{1}, \gamma_{2} / \lambda_{23}, \gamma_{3} / \lambda_{23}\right]\right)\right\}=\mathcal{L}\left(\lambda_{1}, \lambda_{23} ; \boldsymbol{X}\right)
$$

Finally, the estimates of $\lambda_{1}$ and $\lambda_{23}$ can be found setting to zero gradient of $\mathcal{L}\left(\lambda_{1}, \lambda_{23} ; \boldsymbol{X}\right)$ to obtain

$$
\begin{aligned}
\widehat{\lambda}_{1} & =\frac{\gamma_{1}}{K} \\
\widehat{\lambda}_{23} & =\frac{1}{2 K} \sum_{i=2}^{3} \gamma_{i},
\end{aligned}
$$

where $\gamma_{i}, i=1,2,3$, are the eigenvalues of $\boldsymbol{S}$. Gathering the above results, the compressed loglikelihood has the following expression

$$
\mathcal{L}\left(\widehat{\boldsymbol{C}}_{2} ; \boldsymbol{X}\right)=c-K \log \left\{\frac{\gamma_{1}}{K}\right\}-2 K \log \left\{\frac{1}{2 K} \sum_{i=2}^{3} \gamma_{i}\right\}-3 K .
$$

3) Under $H_{3}$ : this case is analogous to the previous hypothesis with the difference that the first two 
eigenvalues of $C$ are equal. Following the same line of reasoning as the previous case, it is possible to show that

$$
\begin{aligned}
\widehat{\boldsymbol{U}} & =\boldsymbol{U}_{S} e^{j \theta} \\
\widehat{\lambda}_{12} & =\frac{1}{2 K} \sum_{i=1}^{2} \gamma_{i} \\
\widehat{\lambda}_{3} & =\frac{\gamma_{3}}{K}
\end{aligned}
$$

where $\lambda_{12}=\lambda_{1}=\lambda_{2}$, and the resulting compressed log-likelihood can be recast as

$$
\mathcal{L}\left(\widehat{\boldsymbol{C}}_{3} ; \boldsymbol{X}\right)=c-2 K \log \left\{\frac{1}{2 K} \sum_{i=1}^{2} \gamma_{i}\right\}-K \log \left\{\frac{\gamma_{3}}{K}\right\}-3 K .
$$

4) Under $H_{4}$ : following the lead of [31], it can be shown that the compressed log-likelihood is

$$
\mathcal{L}\left(\widehat{\boldsymbol{C}}_{4} ; \boldsymbol{X}\right)=c-K \sum_{i=1}^{3} \log \left\{\frac{\gamma_{i}}{K}\right\}-3 K
$$

and the proposition is complete.

Finally, the number unknown parameters under each hypothesis for the homogeneous environment is given by

$$
k_{p}(i)= \begin{cases}1, & \text { if } i=1 \\ 6, & \text { if } i=2, \\ 6, & \text { if } i=3 \\ 9, & \text { if } i=4\end{cases}
$$

and the resulting decision statistics of the MOS rules are

- $H_{1}$ :

$$
-2 c+6 K \log \left\{\frac{\operatorname{tr}[S]}{3 K}\right\}+6 K+\eta
$$

- $\mathrm{H}_{2}$ :

$$
-2 c+2 K \log \left\{\frac{\gamma_{1}}{K}\right\}+4 K \log \left\{\frac{1}{2 K} \sum_{i=2}^{3} \gamma_{i}\right\}+6 K+6 \eta
$$

- $H_{3}$ :

$$
-2 c+4 K \log \left\{\frac{1}{2 K} \sum_{i=1}^{2} \gamma_{i}\right\}+2 K \log \left\{\frac{\gamma_{3}}{K}\right\}+6 K+6 \eta
$$


- $H_{4}$ :

$$
-2 c+2 K \sum_{i=1}^{3} \log \left\{\frac{\gamma_{i}}{K}\right\}+6 K+9 \eta .
$$

The processing steps for the homogeneous environment case are shown in the flow diagram in Figure 5. Starting from the three polarimetric channels a sliding window of $K$ samples is used to extract the polarimetric returns needed to compute the sample covariance matrix $S$. A MOS rule (among AIC/BIC/GIC) is then computed for each hypothesis using (24)-(27) and finally $H_{\hat{h}}$ is selected as:

$$
H_{\hat{h}}=\underset{i=1, \ldots, 4}{\arg \max }\{\operatorname{MOS}(i)\} .
$$

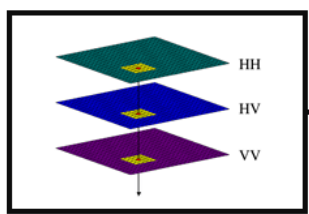

Polarimetric

SAR image
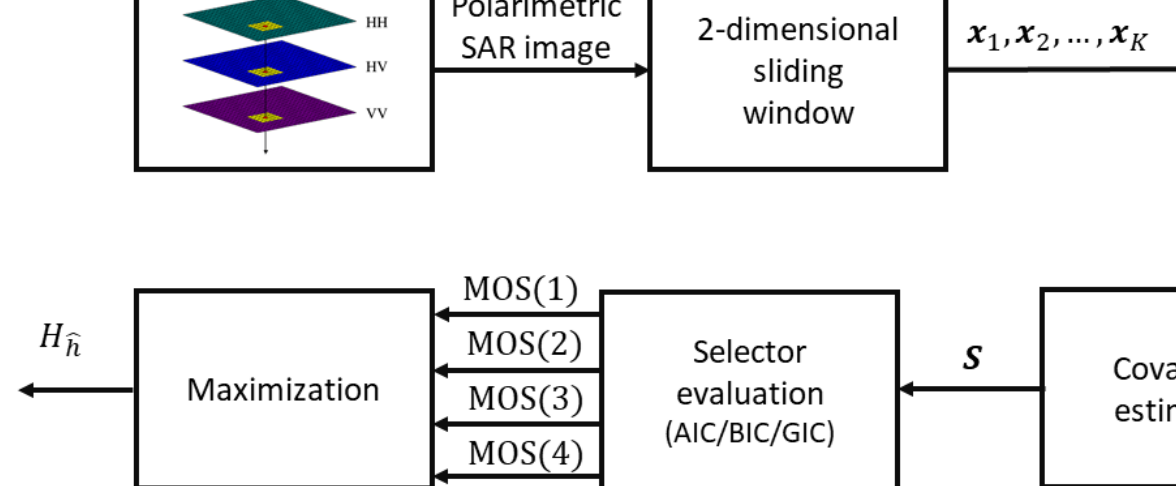

Covariance

estimation

Fig. 5. Processing steps for the selection of the dominant scattering mechanism in homogeneous environment.

\section{B. Heterogeneous Environment}

In this subsection, we assume that the scaling factors, $\sigma_{k}^{2}>0, k=1, \ldots, K$, change due to the different reflectivity strength of each pixel. The dependence on these parameters can be removed exploiting the Principle of Invariance [32], and as shown in [12], [26] the MIS, in this case, is $\boldsymbol{z}_{k}=\boldsymbol{x}_{k} /\left\|\boldsymbol{x}_{k}\right\|$, $k=1, \ldots, K$. Note that $\boldsymbol{z}_{k}, k=1, \ldots, K$, are statistically independent and identically distributed. Indeed, their joint pdf is given by [33]

$$
f\left(\boldsymbol{z}_{1}, \ldots, \boldsymbol{z}_{K} ; \boldsymbol{C}\right)=\frac{1}{[\operatorname{det}(\boldsymbol{C})]^{K}} \prod_{k=1}^{K}\left\{\operatorname{tr}\left(\boldsymbol{C}^{-1} \boldsymbol{S}_{k}\right)\right\}^{-3},
$$

with $\boldsymbol{S}_{k}=\boldsymbol{z}_{k} \boldsymbol{z}_{k}^{\dagger}$. In the following, inference on the eigenvalues of $\boldsymbol{C}$ is performed in the invariant domain. 
Now, in order to obtain the fitting term of the MOS rules, the MLE of $C$ for each hypothesis of (2) is required. However, when $\mathrm{H}_{2}$ or $\mathrm{H}_{3}$ holds, a closed-form expression for the MLE of $\mathrm{C}$ is difficult to obtain. Nevertheless, the MLA paradigm can be still applied to find an estimate of $C$ that somehow optimizes the likelihood function of $\boldsymbol{Z}=\left[\boldsymbol{z}_{1}, \ldots, \boldsymbol{z}_{K}\right]$, whose expression is

$$
l(\boldsymbol{C} ; \boldsymbol{Z})=f\left(\boldsymbol{z}_{1}, \ldots, \boldsymbol{z}_{K} ; \boldsymbol{C}\right) .
$$

The expressions of the required estimates under each hypothesis of problem (2) for the heterogeneous environment are provided by Proposition 1 in [26].

Finally, using the expression of $\mathcal{L}\left(\widehat{\boldsymbol{C}}_{i} ; \boldsymbol{Z}\right)$ in the right-hand side of (3) and that

$$
k_{p}(i)= \begin{cases}0, & \text { if } i=1, \\ 5, & \text { if } i=2, \\ 5, & \text { if } i=3, \\ 8, & \text { if } i=4,\end{cases}
$$

we obtain the following decision statistics for each hypothesis

- $H_{1}$ :

0

- $\mathrm{H}_{2}$ :

$$
2 K \log (\hat{\gamma})+6 \sum_{k=1}^{K} \log \left[\boldsymbol{z}_{k}^{\dagger}\left(\boldsymbol{I}+\left(\frac{1}{\hat{\gamma}}-1\right) \hat{\boldsymbol{u}}_{1} \hat{\boldsymbol{u}}_{1}^{\dagger}\right) \boldsymbol{z}_{k}\right]+5 \eta
$$

- $H_{3}$ :

$$
2 K \log (\hat{\xi})+6 \sum_{k=1}^{K} \log \left[\boldsymbol{z}_{k}^{\dagger}\left(\boldsymbol{I}+\left(\frac{1}{\hat{\xi}}-1\right) \hat{\boldsymbol{u}}_{3} \hat{\boldsymbol{u}}_{3}^{\dagger}\right) \boldsymbol{z}_{k}\right]+5 \eta
$$

- $H_{4}$ :

$$
2 K \log \operatorname{det}(\hat{\boldsymbol{C}})+6 \sum_{k=1}^{K} \log \left(\boldsymbol{z}_{k}^{\dagger} \hat{\boldsymbol{C}}^{-1} \boldsymbol{z}_{k}\right)+8 \eta .
$$

where $\hat{\gamma}=\hat{\lambda}_{1} / \hat{\lambda}_{2} \geq 1$ and $\hat{\xi}=\hat{\lambda}_{3} / \hat{\lambda}_{1}$ with $\hat{\lambda}_{i}$ eigenvalues of $\hat{\boldsymbol{C}} ; \hat{\boldsymbol{u}}_{1}$ and $\hat{\boldsymbol{u}}_{3}$ are the first and the third eigenvector of $\hat{\boldsymbol{C}}$ respectively [26].

The processing steps for the heterogeneous environment case are shown in the flow diagram in Figure 6. Starting from the three polarimetric channels a sliding window of $K$ samples is used to extract the polarimetric returns that are then normalised obtaining $Z$. The normalised observations are then used for 
the recursive estimation of the covariance matrix $\hat{C}$ under the four hypotheses. Then, $Z$ and $\hat{C}$ are used to evaluate equations (31)-(34) in order to obtain the MOSs for the four hypotheses and for one of the criteria (AIC/BIC/GIC). The dominant scattering mechanism is then selected as $H_{\hat{h}}$ namely:

$$
H_{\hat{i}}=\arg \max \{\operatorname{MOS}(i)\}
$$

It is worth to highlight the difference between the processing steps, for the selection of the dominant scattering mechanism, in the homogeneous and heterogeneous environments (this could be easily accomplished comparing Figures 5 and 6). This comparison permits to observe that the heterogeneous case, due to the necessary recursive estimation for the covariance matrix $\hat{C}$, requires a greater computational load with respect to the homogeneous scenario, even though the estimation involves the eigendecomposition for both cases.

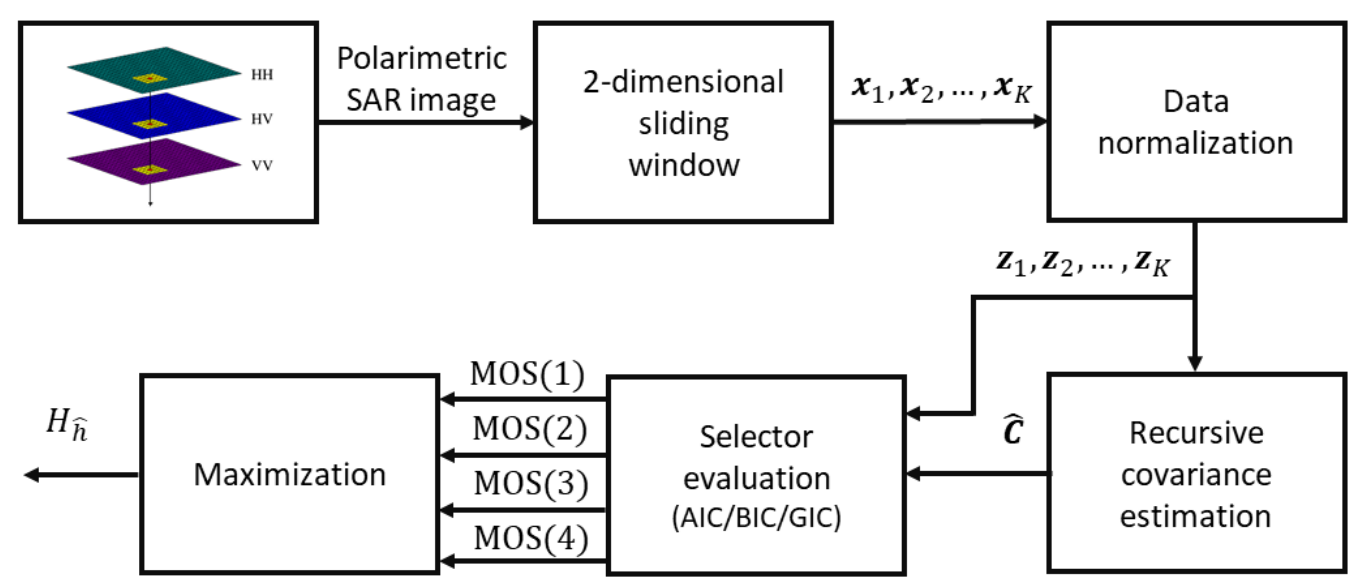

Fig. 6. Processing steps for the selection of the dominant scattering mechanism in heterogeneous environment.

\section{ILlustrative Examples AND Discussion}

The aim of this section is twofold. First, the nominal behavior of the proposed classification architectures is investigated over simulated data adhering the design assumptions. Then, the performance is studied resorting to measured fully-polarimetric SAR data.

\section{A. Analysis on Simulated Data}

In this subsection, the analysis is conducted by means of simulated data and considering the probability of correct classification, $P_{c c}$, as the performance metric. To this end, standard Monte Carlo counting 
techniques are exploited to estimate the $P_{c c}$ over $10^{4}$ independent trials. The nominal covariance matrices associated with the considered four hypotheses are:

- $\boldsymbol{C}_{1}=\operatorname{Diag}\left(\left[\begin{array}{lll}10 & 10 & 10\end{array}\right]^{T}\right)$,

- $\boldsymbol{C}_{2}=\operatorname{Diag}\left(\left[\begin{array}{lll}100 & 1 & 1\end{array}\right]^{T}\right)$,

- $\boldsymbol{C}_{3}=\operatorname{Diag}\left(\left[\begin{array}{lll}100 & 1 & 100\end{array}\right]^{T}\right)$,

- $\boldsymbol{C}_{4}=\operatorname{Diag}\left(\left[\begin{array}{lll}1000 & 100 & 10\end{array}\right]^{T}\right)$.

1) Homogeneous Environment: In the homogeneous case, data are modeled as $N$-dimensional zeromean complex circular Gaussian vectors, with covariance matrix $\boldsymbol{C}_{i}, i=1, \ldots, 4$.

In Figure 7 (a) and (b) the classification histograms are reported for $K=10$ and $K=100$ looks, respectively. Each subplot refers to a specific hypothesis and the classification performance of the AIC, BIC, and GIC with $\rho=3$ are presented ${ }^{2}$. Comparing the two subplots for $K=10$ and $K=100$, it is evident that the performance improves due to a higher number of looks in the evaluation of the MOS rules. In fact, a high number of looks leads to better estimates of the polarimetric covariances. The histograms also highlight that both BIC and GIC exhibit excellent classification capabilities overcoming the AIC which tends to saturate its performance. Moreover, since BIC does not require any additional tuning parameter as for GIC, it stands out as an effective means for eigenvalue pattern classification.

To further corroborate the obtained results, the values of $P_{c c}$ at intermediate looks' number are shown in Figure 8 with focus on the BIC-based estimator. Each line in the plot refers to a different hypothesis. As expected, it is clear that the performances improve as $K$ increases.

Since $P_{c c}$ is a synthetic figure of merit, in Table I, the number of decisions for each one of the considered four hypotheses is also provided as a function of $K$. Inspection of the table makes clear which hypothesis the algorithm chooses in the case of selection error. For instance, the $H_{4}$ hypothesis is never erroneously estimated as $H_{1}$, but for the lowest value of $K$, it is erroneously classified as $H_{2}$ and $H_{3}, 568$ and 413 times, respectively, over a total of $10^{4}$ trials.

\footnotetext{
${ }^{2}$ The choice of the GIC parameter comes from the fact that it returns the best performance with respect to the cases where $1 \leq \rho \leq 3$. Moreover, values of $\rho$ greater than 3 could lead to worse classification performance since the penalty term becomes more and more dominant with respect to the fitting term.
} 
(a) $K=10$
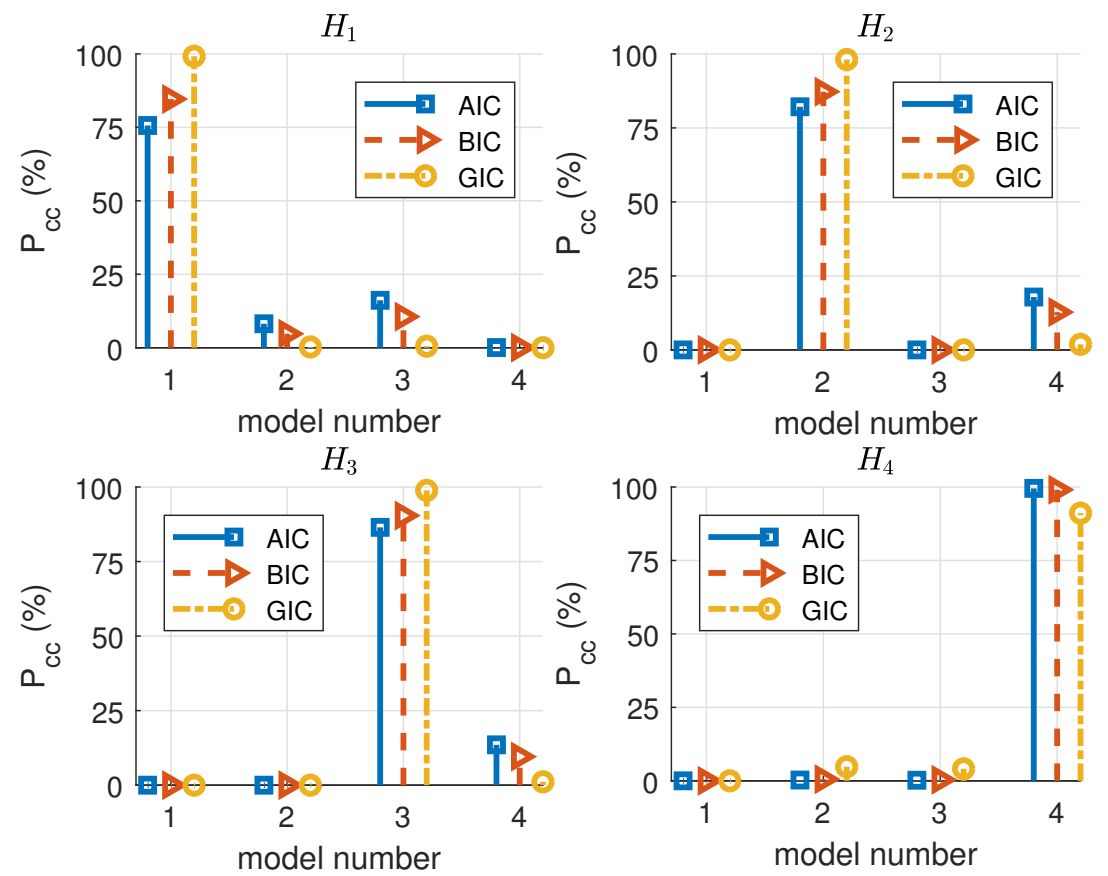

(b) $K=100$
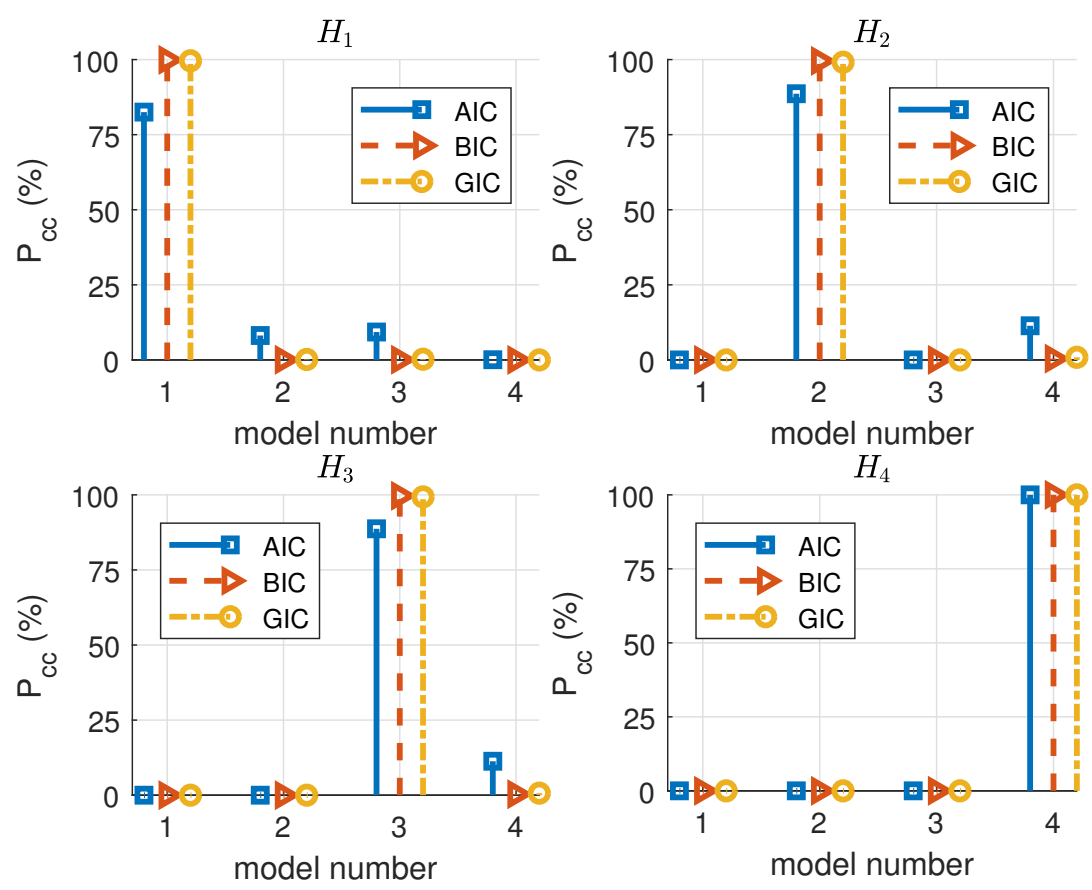

Fig. 7. Homogeneous case performance analysis: $P_{c c}(\%)$ of the three MOS rules for a simulated scenario with $K=10$ (a) and $\mathrm{K}=100$ (b) looks and $10^{4}$ Monte Carlo trials. Subfigures from left to right and top to bottom refer to the different eigenvalues patterns. 


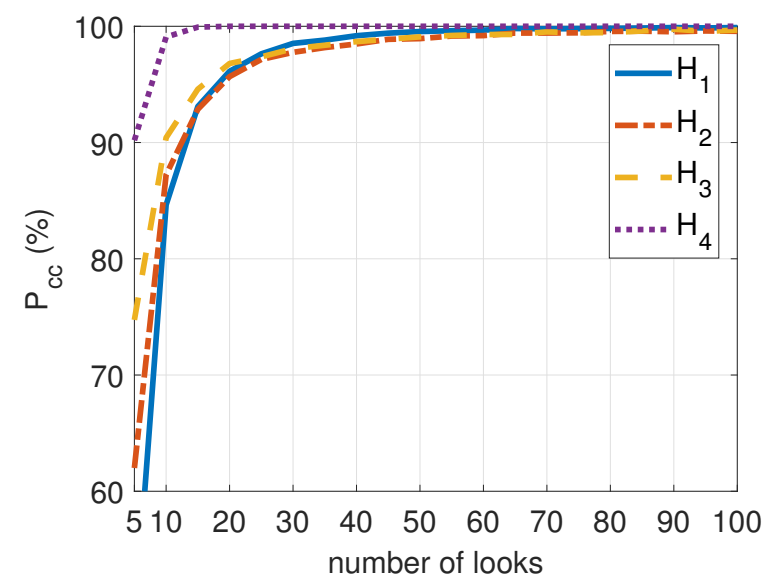

Fig. 8. Homogeneous case performance analysis: $P_{c c}(\%)$ versus the number of looks $K$ for the BIC selector, and $10^{4}$ Monte Carlo trials.

TABLE I

NUMBER OF DECISIONS FOR EACH HYPOTHESIS IN THE HOMOGENEOUS SIMULATED SCENARIO OF FIGURE 8 VERSUS THE NUMBER OF LOOKS $K$ FOR THE BIC SELECTOR AND $10^{4}$ TRIALS.

\begin{tabular}{|c|c|c|c|c|c|c|c|c|c|c|c|}
\hline \multirow{2}{*}{ true } & \multirow{2}{*}{ estimated } & \multicolumn{10}{|c|}{$K$} \\
\hline & & 5 & 15 & 25 & 35 & 45 & 55 & 65 & 75 & 85 & 95 \\
\hline \multirow{4}{*}{$H_{1}$} & $H_{\hat{1}}$ & 4806 & 9310 & 9763 & 9881 & 9941 & 9962 & 9981 & 9980 & 9985 & 9986 \\
\hline & $H_{\hat{2}}$ & 1292 & 224 & 93 & 45 & 30 & 22 & 9 & 7 & 6 & 1 \\
\hline & $H_{\hat{3}}$ & 3754 & 466 & 144 & 74 & 29 & 16 & 10 & 13 & 9 & 13 \\
\hline & $H_{\hat{4}}$ & 148 & 0 & 0 & 0 & 0 & 0 & 0 & 0 & 0 & 0 \\
\hline \multirow{4}{*}{$H_{2}$} & $\overline{H_{\hat{1}}}$ & 0 & 0 & 0 & 0 & 0 & 0 & 0 & 0 & 0 & 0 \\
\hline & $H_{\hat{2}}$ & 6200 & 9286 & 9715 & 9817 & 9888 & 9916 & 9942 & 9944 & 9958 & 9960 \\
\hline & $H_{\hat{3}}$ & 2 & 0 & 0 & 0 & 0 & 0 & 0 & 0 & 0 & 0 \\
\hline & $\tilde{H_{\hat{4}}}$ & 3798 & 714 & 285 & 183 & 112 & 84 & 58 & 56 & 42 & 40 \\
\hline \multirow{4}{*}{$H_{3}$} & $H_{\hat{1}}$ & 0 & 0 & 0 & 0 & 0 & 0 & 0 & 0 & 0 & 0 \\
\hline & $H_{\hat{2}}$ & 2 & 0 & 0 & 0 & 0 & 0 & 0 & 0 & 0 & 0 \\
\hline & $H_{\hat{3}}$ & 7474 & 9459 & 9737 & 9837 & 9889 & 9921 & 9930 & 9944 & 9960 & 9956 \\
\hline & $H_{\hat{4}}$ & 2524 & 541 & 263 & 163 & 111 & 79 & 70 & 56 & 40 & 44 \\
\hline \multirow{4}{*}{$H_{4}$} & $\overline{H_{\hat{1}}}$ & 0 & 0 & 0 & 0 & 0 & 0 & 0 & 0 & 0 & 0 \\
\hline & $H_{\hat{2}}$ & 568 & 5 & 0 & 0 & 0 & 0 & 0 & 0 & 0 & 0 \\
\hline & $H_{\hat{3}}$ & 413 & 2 & 0 & 0 & 0 & 0 & 0 & 0 & 0 & 0 \\
\hline & $H_{\hat{4}}$ & 9019 & 9993 & 10000 & 10000 & 10000 & 10000 & 10000 & 10000 & 10000 & 10000 \\
\hline
\end{tabular}


2) Heterogeneous Environment: Data are modeled as $N$-dimensional ( $N=3$, in this case) Spherically Invariant Random Vectors (SIRVs) [34], namely

$$
\boldsymbol{x}_{k}=\sqrt{\tau_{k}} \boldsymbol{g}_{k}, \quad k=1, \ldots, K,
$$

where $\boldsymbol{g}_{k}$ s are statistically independent $N$-dimensional zero-mean complex circular Gaussian vectors with covariance matrix $\boldsymbol{C}_{i}, i=1, \ldots, 4$. Moreover, $\tau_{1}, \ldots, \tau_{K}$, referred to as textures, are statistically independent (also of $\boldsymbol{x}_{k}$ ) positive real random variables obeying the Gamma distribution with scale and shape parameters $\mu$ and $\nu>0$, respectively (the considered setting assumes $\mu=1 / \nu$ to have a Gamma distribution with unit mean). In what follows, it is assumed $\nu=2$. Furthermore, the number of iterations used to estimate the covariance structure is 5 , that ensures a satisfactory convergence level as proved in [12].

Figures 9 (a) and (b) show the classification histograms for $K=10$ and $K=100$ looks, respectively. Again, as for the previous case, it turns out that a performance gain is achieved considering a higher number of looks, and, also that both BIC and GIC performances overcome the AIC, which suffers performance saturation effects instead.

In the next analysis, the $P_{c c}$ is plotted versus the number of snapshots. The simulating environment is the same as in Figure 9, but for different values of the shape parameter, i.e. $\nu=0.5,1,2,5$, and with focus on the BIC-based estimator. Again, each subfigure refers to a different hypothesis for the dominant eigenvalues. From this analysis, it is clear that the classification performance is insensitive to variations of $\nu$, due to the fact that the architectures for heterogeneous environment work in the invariant domain. Moreover, better performance can be obtained when $K$ increases, since the covariance estimate becomes more and more reliable.

As before, in Table II, the number of decisions for each hypothesis against $K$ is also provided for the scenario of Figure 10. without loss of generality, the results in Table II, refers to the case $\nu=2$ since the performance is insensitive to the scale and shape parameters. Again, observing the values reported in the table, it is evident the behavior of the algorithm also in terms of erroneous classification. 
(a) $\mathrm{K}=10$
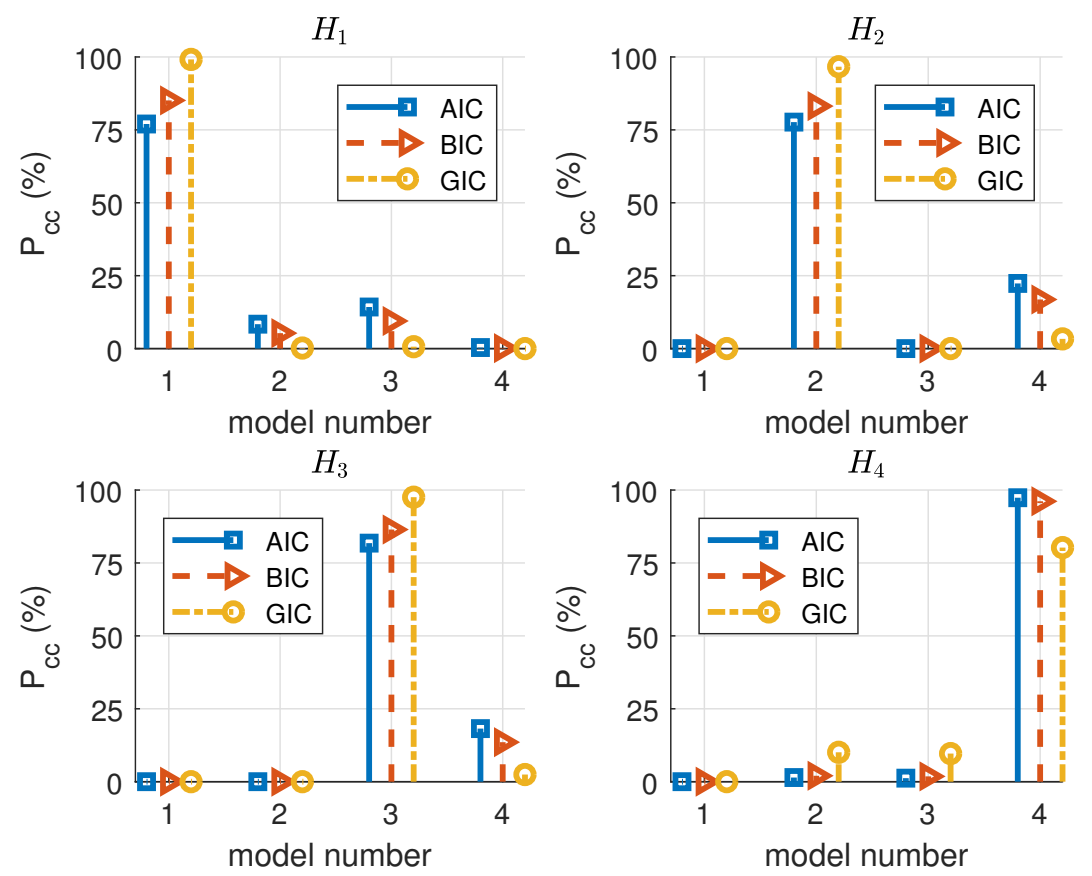

(b) $\mathrm{K}=100$
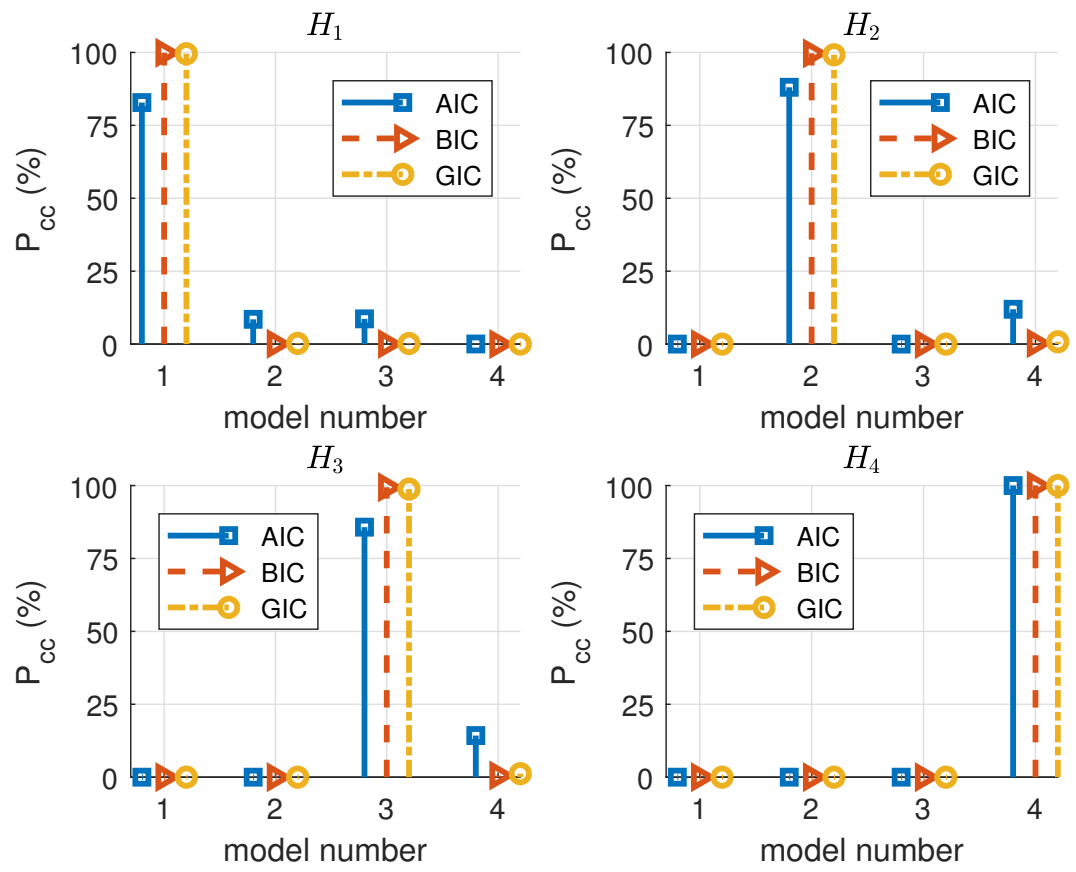

Fig. 9. Heterogeneous case performance analysis: $P_{c c}(\%)$ of the three MOSs for a simulated scenario with $K=10$ (subfigures a) and $K=100$ (subfigures b) looks and $10^{4}$ Monte Carlo trials. Figures from left to right and top to bottom refer to the different eigenvalues patterns. 

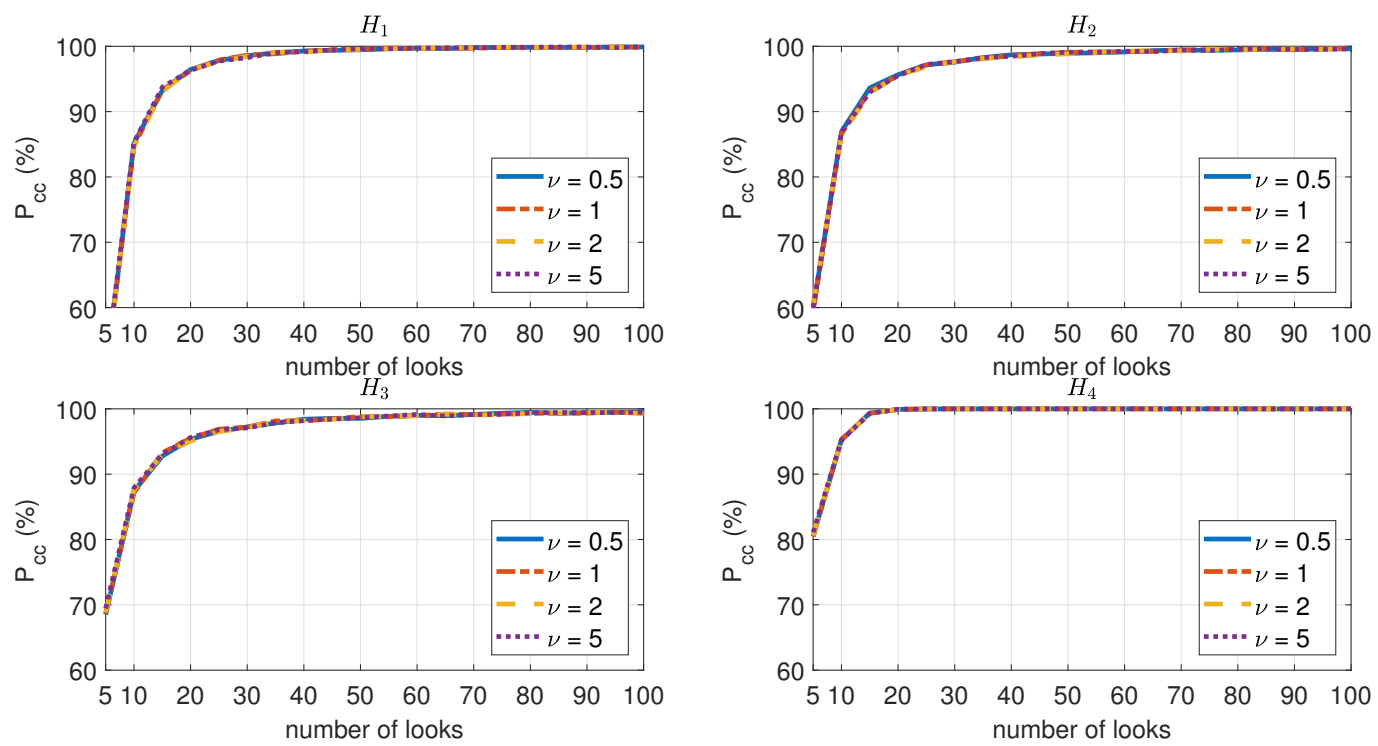

Fig. 10. Heterogeneous case performance analysis: $P_{c c}(\%)$ versus the number of looks $K$ for the BIC selector in a SIRV environment for different scale parameter values and $10^{4}$ Monte Carlo trials.

TABLE II

NUMBER OF DECISIONS FOR EACH HYPOTHESIS IN THE HETEROGENEOUS SIMULATED SCENARIO OF FIGURE 10 VERSUS THE NUMBER OF LOOKS $K$, FOR THE BIC SELECTOR AND $10^{4}$ TRIALS.

\begin{tabular}{|c|c|c|c|c|c|c|c|c|c|c|c|}
\hline \multirow{2}{*}{ true } & \multirow{2}{*}{ estimated } & \multicolumn{10}{|c|}{$K$} \\
\hline & & 5 & 15 & 25 & 35 & 45 & 55 & 65 & 75 & 85 & 95 \\
\hline \multirow{4}{*}{$H_{1}$} & $\overline{H_{\hat{1}}}$ & 5145 & 9349 & 9782 & 9891 & 9942 & 9958 & 9972 & 9985 & 9986 & 9987 \\
\hline & $H_{\hat{2}}$ & 1345 & 227 & 94 & 46 & 19 & 19 & 13 & 9 & 8 & 5 \\
\hline & $H_{\hat{3}}$ & 3121 & 423 & 124 & 63 & 39 & 23 & 15 & 6 & 6 & 8 \\
\hline & $\frac{3}{H_{\hat{4}}}$ & 387 & 1 & 0 & 0 & 0 & 0 & 0 & 0 & 0 & 0 \\
\hline \multirow{4}{*}{$H_{2}$} & $H_{\hat{1}}$ & 0 & 0 & 0 & 0 & 0 & 0 & 0 & 0 & 0 & 0 \\
\hline & $H_{\hat{2}}$ & 5592 & 9059 & 9576 & 9734 & 9813 & 9853 & 9902 & 9924 & 9923 & 9937 \\
\hline & $H_{\hat{3}}$ & 3 & 0 & 0 & 0 & 0 & 0 & 0 & 0 & 0 & 0 \\
\hline & $\frac{3}{H_{\hat{4}}}$ & 4405 & 941 & 424 & 266 & 187 & 147 & 98 & 76 & 77 & 63 \\
\hline \multirow{4}{*}{$H_{3}$} & $\overline{H_{\hat{1}}}$ & 0 & 0 & 0 & 0 & 0 & 0 & 0 & 0 & 0 & 0 \\
\hline & $H_{\hat{2}}$ & 16 & 0 & 0 & 0 & 0 & 0 & 0 & 0 & 0 & 0 \\
\hline & $H_{\hat{3}}$ & 6721 & 9268 & 9629 & 9766 & 9820 & 9865 & 9892 & 9919 & 9933 & 9932 \\
\hline & $H_{\hat{4}}$ & 3263 & 732 & 371 & 234 & 180 & 135 & 108 & 81 & 67 & 68 \\
\hline \multirow{4}{*}{$H_{4}$} & $\overline{H_{\hat{1}}}$ & 2 & 0 & 0 & 0 & 0 & 0 & 0 & 0 & 0 & 0 \\
\hline & $H_{\hat{2}}$ & 831 & 21 & 0 & 0 & 0 & 0 & 0 & 0 & 0 & 0 \\
\hline & $H_{\hat{3}}$ & 825 & 24 & 1 & 0 & 0 & 0 & 0 & 0 & 0 & 0 \\
\hline & $H_{\hat{4}}$ & 8342 & 9955 & 9999 & 10000 & 10000 & 10000 & 10000 & 10000 & 10000 & 10000 \\
\hline
\end{tabular}




\section{B. Analysis on Measured SAR Data}

In this subsection, the classification capabilities of the proposed methods are tested over a C-band $(5.405 \mathrm{GHz})$ polarimetric dataset ${ }^{3}$, acquired by the multiple polarization modes spaceborne SAR system on-board the RADARSAT-2, launched in December 2007. Particularly, for the case at hand, the fine quad-Polarization mode (Fine Quad-Pol) Single Look Complex (SLC) product data, characterized by a nominal resolution of $5.2 \mathrm{~m}$ (range) $\times 7.7 \mathrm{~m}$ (azimuth), have been used [35].

The image, acquired on April 2008, represents a scene of the Vancouver area (western Canada) containing a mixed urban, vegetation and water scene; the corresponding optical image, drawn from SNAP - ESA Sentinel Application Platform [36], is shown in Figure 11 (the pixels of the quad-Pol SLC RADARSAT-2 data are highlighted with a rectangular red box in the image). In Table III, the main information regarding the SAR acquisition is summarized.

As it is possible to notice from Figure 11, this geographic location represents a valuable test for the proposed procedure, since it offers varied terrain types ranging from the rugged mountains of the north of Vancouver, to the flat, agricultural lands of the Fraser River Delta.

Given the satisfying performance of BIC on simulated data, its behavior is investigated here considering a sliding window of $K=25$ pixels and 5 iterations for the covariance structure estimation. In Figure 12, the classification results and the Entropy Map (EM) ${ }^{4}$ are shown [10], [25]. Note that the latter ranges from 0 (only one nonzero eigenvalue) to 1 (three equal nonzero eigenvalues). Figures 12(a) and (b) are the results achieved using the homogeneous and heterogeneous classification rules, respectively. In both subfigures, a specific color is associated with each of the possible classification outcomes according to the following scheme: black for $H_{1}$, red for $H_{2}$, blue for $H_{3}$, yellow for $H_{4}$.

The comparison between Figures 11 and 12 highlights the effectiveness of the proposed approach. In fact, the following considerations can help to interpret the achieved results: $H_{1}$ means that three scattering mechanisms sharing the same strength are identified; in the case of $H_{2}$ two dominant scattering mechanisms with different reflectivity are present; $H_{3}$ can be interpreted as the action of two dominant scattering mechanisms sharing the same power; and, for $H_{4}$ there are no dominant structures that tend to favor one polarization rather than another.

With this in mind, as expected, the forests are classified as the first three hypotheses and this behavior

\footnotetext{
${ }^{3}$ Sample data for RADARSAT-2 products supplied by MacDonald Dettwiler and Associates (MDA) can be found at: https://mdacorporation.com/geospatial/international/satellites/RADARSAT-2/sample-data

${ }^{4}$ Note that the EM allows to measure the amount of effective scattering processes embedded in the polarimetric covariance matrix without providing any additional information about the relationship among the polarimetric eigenvalues.
} 
can be explained by the fact that, generally speaking, in forest areas, three kinds of scattering mechanisms take place due to the presence of a mixture of vertical trunks, vertical and horizontal branches, and obliques structures. Inspection of the EM confirms this result since it returns values greater than 0.5 in those areas. The urban zones are classified as $H_{3}$ due to the fact that buildings mainly respond approximately with the same strength to both horizontal and vertical polarizations. The corresponding values of the EM, which belong to $[0.5,0.9]$, corroborate this result. Finally, most of pixels associated with the water and the cultivated fields are classified as $H_{4}$ since, in this case, there are no dominant structures that tend to favor one polarization rather than another. This behavior is in accordance with the values of the EM in the same area.

TABLE III

METADATA INFORMATION REGARDING THE RADARSAT-2 DATASET

\begin{tabular}{|l|r|}
\hline Product ID & RS2-SLC-FQ2-DES-15-Apr-2008_14.38-PDS_05116980 \\
\hline Product Type & SLC \\
\hline Acquisition Mode & Fine Quad-Pol \\
\hline Time of orbit & 15-APR-2008 $14: 38: 07.763803$ \\
\hline Pass & Descending \\
\hline Near incidence angle & $19.78838974574827 \mathrm{deg}$ \\
\hline Far incidence angle & $21.826735667462618 \mathrm{deg}$ \\
\hline Range sample spacing & $4.73307896 \mathrm{~m}$ \\
\hline Azimuth sample spacing & $4.87164879 \mathrm{~m}$ \\
\hline Pulse Repetition Frequency & $2737.718017578125 \mathrm{~Hz}$ \\
\hline Radar Frequency & $5404.999242769673 \mathrm{MHz}$ \\
\hline
\end{tabular}

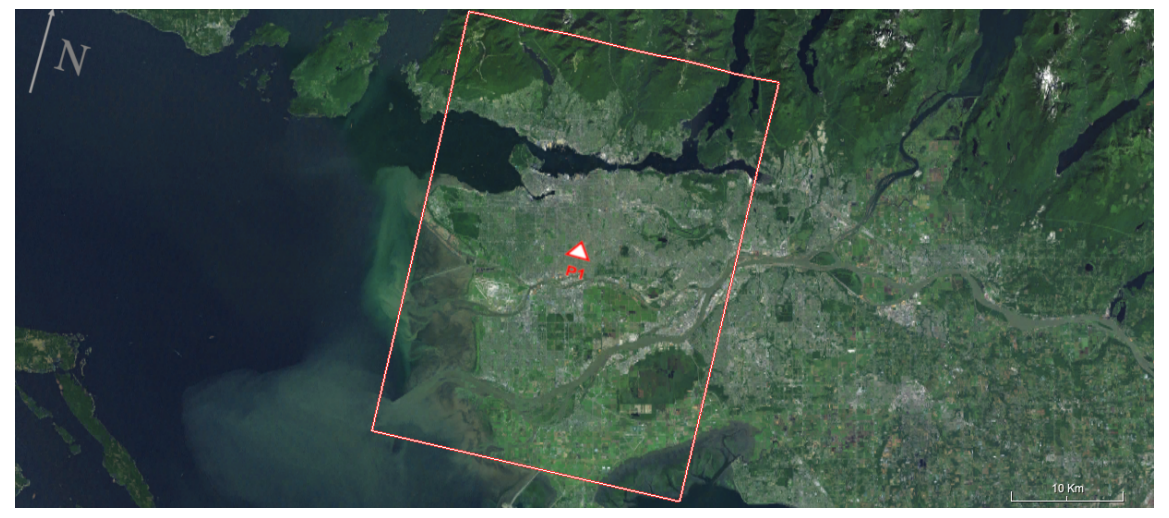

Fig. 11. Optical image of the observed scene (drawn from SNAP - ESA Sentinel Application Platform [36]). The quad-Pol SLC RADARSAT-2 image is highlighted with a rectangular red box and the point P1 is geolocated at the coordinates Datum World Geodetic System (WGS)-84: 49¹3'17.0400”N, 12304'16.6800”W. 

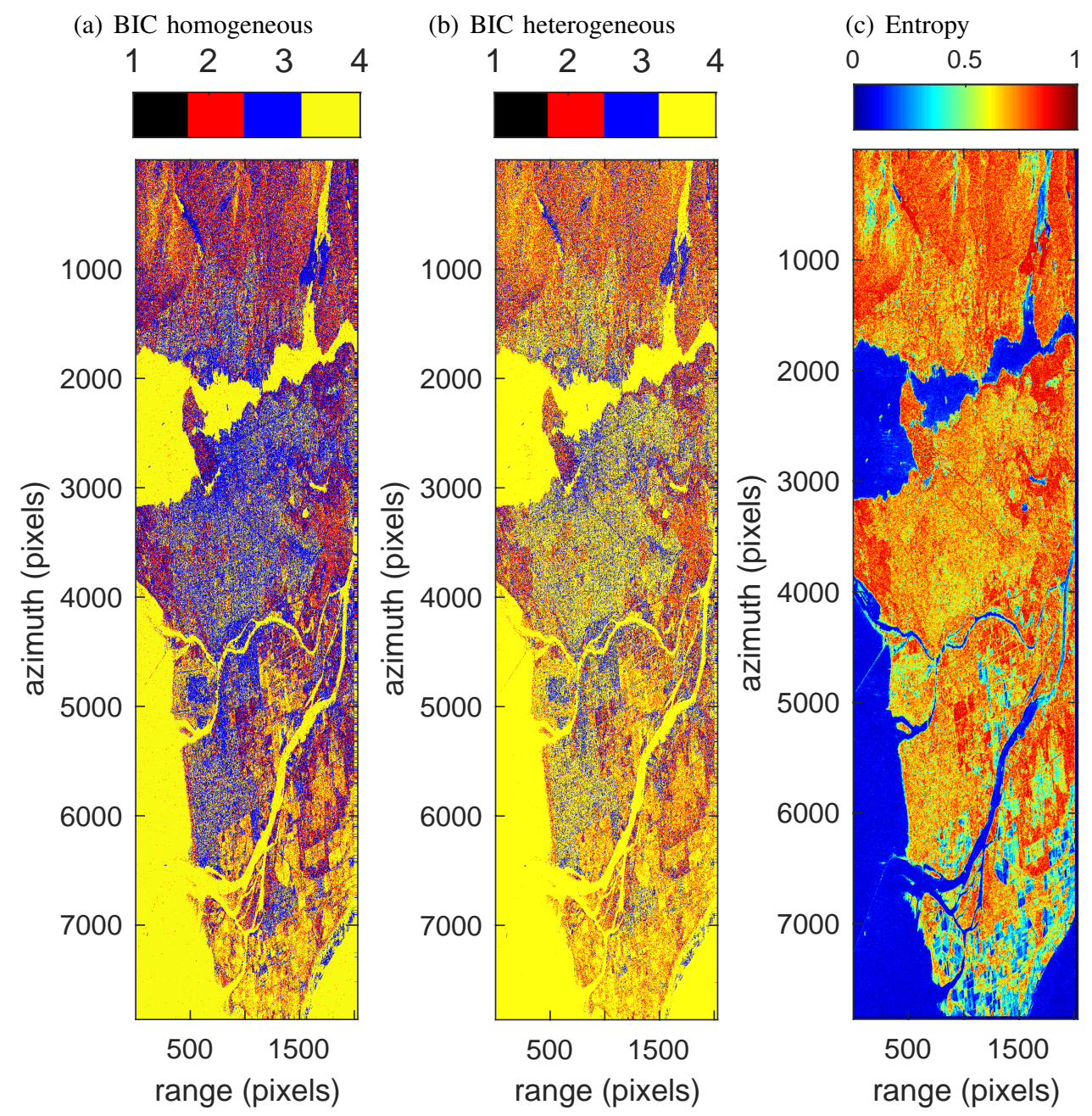

Fig. 12. Detected dominant eigenvalues (subplot a and b) within the reference image with the BIC-based selector, $K=25$. Entropy map of the same scene (subplot c).

\section{CONCLUSIONS}

In this work, we have analyzed the problem of automatic classification of the dominant scattering mechanisms associated with the pixels of polarimetric SAR images. At the design stage, we have assumed that the polarimetric image pixels locally share the same covariance except for possible scaling factors (homogeneous and heterogeneous environment). Then, the MOS rules have been applied to the original data in the case of homogeneous environment, whereas the invariant domain has been exploited in the heterogeneous case to get rid of the power variations. The performance analysis, conducted on both simulated and measured data, have highlighted the effectiveness of the proposed classification rules. Particularly, results on Monte Carlo simulations highlighted that BIC and GIC-based estimators can 
achieve better performance with respect to the AIC. Thus, since BIC does not require the additional parameter as for the GIC, it represents the best solution at least for the considered parameter values. Remarkably, the analysis on real polarimetric SAR data has further confirmed the classification capabilities of the proposed solutions which arise as an effective means to build up automatic classification systems.

Future research might consider further investigation using SAR data with different radar frequency and the identification of the specific polarimetric channel which generates the dominant eigenvalue.

\section{ACKNOWLEDGEMENTS}

The authors would like to thank the Canadian Space Agency (CSA) and the MacDonald Dettwiler and Associates (MDA) for providing RADARSAT-2 fine quad-polarization mode single-look complex SAR data.

They would also to thank the Editor and the Reviewers for careful reading and helpful comments.

\section{REFERENCES}

[1] D. Giuli, "Polarization Diversity in Radars," Proceedings of the IEEE, vol. 74, no. 2, pp. 245-269, February 1986.

[2] L. M. Novak, M. B. Sechtin, and M. J. Cardullo, "Studies of target detection algorithms that use polarimetric radar data," IEEE Transactions on Aerospace and Electronic Systems, vol. 25, no. 2, pp. 150-165, 1989.

[3] A. De Maio, D. Orlando, L. Pallotta, and C. Clemente, “A Multifamily GLRT for Oil Spill Detection,” IEEE Trans. on Geoscience and Remote Sensing, vol. 55, no. 1, pp. 63-79, January 2017.

[4] C. Hao, S. Gazor, X. Ma, S. Yan, C. Hou, and D. Orlando, "Polarimetric detection and range estimation of a point-like target," IEEE Transactions on Aerospace and Electronic Systems, vol. 52, no. 2, pp. 603-616, April 2016.

[5] F. Filippini, F. Colone, D. Cristallini, and G. Bournaka, "Experimental results of polarimetric detection schemes for DVB-T-based passive radar," IET Radar, Sonar Navigation, vol. 11, no. 6, pp. 883-891, 2017.

[6] M. C. Dobson, F. T. Ulaby, L. E. Pierce, T. L. Sharik, K. M. Bergen, J. Kellndorfer, J. R. Kendra, E. Li, Y. C. Lin, A. Nashashibi, K. Sarabandi, and P. Siqueira, "Estimation of forest biophysical characteristics in Northern Michigan with SIR-C/X-SAR,” IEEE Transactions on Geoscience and Remote Sensing, vol. 33, no. 4, pp. 877-895, July 1995.

[7] J. M. Lopez-Sanchez, S. R. Cloude, and J. D. Ballester-Berman, "Rice Phenology Monitoring by Means of SAR Polarimetry at X-Band," IEEE Transactions on Geoscience and Remote Sensing, vol. 50, no. 7, pp. 2695-2709, July 2012.

[8] Jiancheng Shi and J. Dozier, "Inferring snow wetness using C-band data from SIR-C's polarimetric synthetic aperture radar," IEEE Transactions on Geoscience and Remote Sensing, vol. 33, no. 4, pp. 905-914, July 1995.

[9] L. Pallotta, C. Clemente, A. De Maio, and J. J. Soraghan, "Detecting Covariance Symmetries in Polarimetric SAR Images," IEEE Transactions on Geoscience and Remote Sensing, vol. 55, no. 1, pp. 80-95, Jan 2017.

[10] J. S. Lee and E. Pottier, Polarimetric Radar Imaging: From Basics to Applications, CRC Press, 2009.

[11] L. Ferro-Famil and E. Pottier (Editor in Chief J. A. Kong), Progress In Electromagnetics Research, vol. 24, chapter Dual Frequency Polarimetric SAR Data Classification and Analysis, pp. 251-276, New York Elsevier, 2001. 
[12] L. Pallotta, A. De Maio, and D. Orlando, “A Robust Framework for Covariance Classification in Heterogeneous Polarimetric SAR Images and its Application to L-Band Data," IEEE Transactions on Geoscience and Remote Sensing, vol. 57, no. 1, pp. 104-119, January 2019.

[13] S. Tahraoui, C. Clemente, L. Pallotta, J. J. Soraghan, and M. Ouarzeddine, "Covariance Symmetries Detection in PolInSAR Data," IEEE Transactions on Geoscience and Remote Sensing, vol. 56, no. 12, pp. 6927-6939, Dec 2018.

[14] Alberto Moreira, Pau Prats-Iraola, Marwan Younis, Gerhard Krieger, Irena Hajnsek, and Konstantinos P. Papathanassiou, “A Tutorial on Synthetic Aperture Radar,” IEEE Geoscience and Remote Sensing Magazine, vol. 1, no. 1, pp. 6-43, 2013.

[15] G. G. Lemoine, G. F. De Grandi, and A. J. Sieber, "Polarimetric Contrast Classification of Agricultural Fields using MAESTRO1 AIRSAR Data," International Journal on Remote Sensing, vol. 15, no. 14, pp. 2851-2869, March 1994.

[16] J. S. Lee, K. P. Papathanassiou, I. Hajnsek, T. Mette, M. R. Grunes, T. L. Ainsworth, and L. Ferro-Famil, “Applying Polarimetric SAR Interferometric Data for Forest Classification,” in IEEE International Geoscience and Remote Sensing Symposium (IGARSS). IEEE, 2005, vol. 7.

[17] L. Zhang, B. Zou, J. Zhang, and Y. Zhang, "Classification of Polarimetric SAR Image Based on Support Vector Machine Using Multiple-Component Scattering Model and Texture Features,” EURASIP Journal on Advances in Signal Processing, vol. 2010, no. 1, pp. 1-9, December 2009.

[18] M. Dabboor and M. Shokr, "A New Likelihood Ratio for Supervised Classification of Fully Polarimetric SAR Data: An Application for Sea Ice Type Mapping,” ISPRS Journal of Photogrammetry and Remote Sensing, vol. 84, pp. 1-11, 2013.

[19] S. Uhlmann and S. Kiranyaz, "Evaluation of Classifiers for Polarimetric SAR Classification," in IEEE International Geoscience and Remote Sensing Symposium (IGARSS). IEEE, 2013, pp. 775-778.

[20] J. J. Van Zyl, "Unsupervised Classification of Scattering Behavior Using Radar Polarimetry Data," IEEE Trans. on Geoscience and Remote Sensing, vol. 27, no. 1, pp. 36-45, January 1989.

[21] J. S. Lee, M. R. Grunes, T. L. Ainsworth, L. J. Du, D. L. Schuler, and S. R. Cloude, "Unsupervised Classification Using Polarimetric Decomposition and the Complex Wishart Classifier," IEEE Trans. on Geoscience and Remote Sensing, vol. 37, no. 5, pp. 2249-2258, September 1999.

[22] L. Ferro-Famil, E. Pottier, and J. S. Lee, "Unsupervised Classification of Multifrequency and Fully Polarimetric SAR Images Based on the H/A/Alpha-Wishart Classifier," IEEE Trans. on Geoscience and Remote Sensing, vol. 39, no. 11, pp. 2332-2342, November 2001.

[23] J. S. Lee, M. R. Grunes, E. Pottier, and L. Ferro-Famil, "Unsupervised Terrain Classification Preserving Polarimetric Scattering Characteristics," IEEE Trans. on Geoscience and Remote Sensing, vol. 42, no. 4, pp. 722-731, April 2004.

[24] N. Zhong, W. Yang, A. Cherian, X. Yang, G. S. Xia, and M. Liao, "Unsupervised Classification of Polarimetric SAR Images via Riemannian Sparse Coding,” IEEE Trans. on Geoscience and Remote Sensing, vol. 55, no. 9, pp. 5381-5390, September 2017.

[25] S. R. Cloude and E. Pottier, "An Entropy Based Classification Scheme for Land Applications of Polarimetric SAR,” IEEE Trans. on Geoscience and Remote Sensing, vol. 35, no. 1, pp. 68-78, January 1997.

[26] L. Pallotta and D. Orlando, "Polarimetric Covariance Eigenvalues Classification in SAR Images," IEEE Geoscience and Remote Sensing Letters, pp. 1-5, 2018.

[27] S. M. Kay, Fundamentals of Statistical Signal Processing: Estimation Theory, vol. 1, Prentice Hall, 1993.

[28] S. M. Kay, “The Multifamily Likelihood Ratio Test for Multiple Signal Model Detection,” IEEE Signal Processing Letters, vol. 12, no. 5, pp. 369-371, May 2005. 
[29] P. Stoica and Y. Selen, "Model-order selection: A review of information criterion rules," IEEE Signal Processing Magazine, vol. 21, no. 4, pp. 36-47, 2004.

[30] L. Mirsky, “On the trace of matrix products," Mathematische Nachrichten, vol. 20, pp. 171-174, 1959.

[31] E. J. Kelly, “An adaptive detection algorithm," IEEE Transactions on Aerospace and Electronic Systems, , no. 2, pp. 115-127, 1986.

[32] E. L. Lehmann, Testing Statistical Hypotheses, Springer-Verlag, New York, USA, 2nd ed. edition, 1986.

[33] E. Ollila, D. E. Tyler, V. Koivunen, and H. V. Poor, "Complex elliptically symmetric distributions: Survey, new results and applications," IEEE Transactions on Signal Processing, vol. 60, no. 11, pp. 5597-5625, 2012.

[34] E. Conte and M. Longo, "Characterisation of Radar Clutter as a Spherically Invariant Random Process," IEE Proceedings Communications, Radar and Signal Processing, vol. 134, no. 2, pp. 191-197, April 1987.

[35] "RADARSAT - 2 PRODUCT DESCRIPTION," RN-SP-52-1238 Issue 1/14: September 10, 2018, available at: https://mdacorporation.com.

[36] “SNAP - ESA Sentinel Application Platform v6.0,” http://step.esa.int. 